\title{
Passive Wireless Strain and pH Sensing Using Carbon Nanotube-Gold Nanocomposite Thin Films
}

\author{
Kenneth J. Loh ${ }^{\mathrm{a}}$, Jerome P. Lynch ${ }^{* a, b}$, and Nicholas A. Kotov ${ }^{\mathrm{c}}$ \\ ${ }^{a}$ University of Michigan, Dept. of Civil \& Environmental Engineering; \\ ${ }^{b}$ University of Michigan, Dept. of Electrical Engineering \& Computer Science; \\ ${ }^{c}$ University of Michigan, Dept. of Chemical Engineering, Ann Arbor, MI 48109.
}

\begin{abstract}
The recent development of wireless sensors for structural health monitoring has revealed their strong dependency on portable, limited battery supplies. Unlike current wireless sensors, passive radio frequency identification (RFID) systems based on inductive coupling can wirelessly receive power from a portable reader while transmitting collected data back. In this paper, preliminary results of a novel inductively coupled strain and corrosion sensor based upon material fabrication techniques from the nanotechnology field are presented. By varying polyelectrolyte species during a layer-by-layer fabrication process, carbon nanotube-polyelectrolyte multilayer thin film sensors sensitive to different mechanical (e.g. strain) and chemical (e.g. pH) stimuli can be produced. Validation studies conducted with different carbon nanotube thin films designed as either strain or $\mathrm{pH}$ sensors reveal high sensitivity and linear performance. When coupled with a copper inductive coil antenna, resulting RFID-based sensors exhibit wirelessly readable changes in resonant frequency and bandwidth. Furthermore, a carbon nanotube-gold nanocomposite thin film is fabricated and patterned into a highly conductive coil structure to realize a novel thin film inductive antenna. Preliminary results indicate that nanotube-gold nanocomposites exhibit resonance conditions, holding great promise for future RFID applications.
\end{abstract}

Keywords: Carbon nanotube, gold nanoparticles, layer-by-layer assembly, pH, RFID, strain, wireless.

\section{INTRODUCTION}

Over the lifespan of civil infrastructures (namely bridges, buildings, pipelines, among many others), unanticipated extreme loading (e.g. earthquakes) and harsh environmental conditions (e.g. deicing salt on roadways) can adversely affect long-term structural integrity and performance. As a result, researchers in the structural health monitoring (SHM) community have utilized tethered sensor networks to measure structural responses that can be used to identify structural deterioration. While traditional cabled monitoring systems are reliable, extensive cabling leads to high capital, installation, and maintenance costs. For instance, a recent instrumentation on the Tsing Ma suspension bridge in Hong Kong (2001) intended to monitor bridge behavior to strong wind and seismic loading costs $\$ 27,000$ per sensing channel [1]. Such high costs prevent many users from installing dense sensor networks that would be needed to identify local damage. In recent years, a variety of low cost academic and commercial wireless sensor prototypes have been developed in hopes to outperform traditional tethered monitoring systems [2]. By providing reliable wireless data communication and embedded computing power, many wireless sensor prototypes have been validated in the laboratory and in the field to reveal performance levels just as good, if not better, than cabled systems [3]. Unfortunately, one significant disadvantage of wireless sensors is that they typically require portable power supplies (i.e. batteries) coupled with them. Nevertheless, their lower costs permit dense sensor network installation to allow the transitioning from global-based (e.g. modal analysis) to component-level monitoring.

Since wireless sensors suffer from finite power constraints, many researchers have developed low-cost wireless sensors based on inductive coupling, also known as radio frequency identification (RFID) [2]. In particular, RFID sensors for monitoring strain and corrosion environments $(\mathrm{pH})$ have been proposed. Both types of sensors are monitoring the structure at the local-level with strain correlated to some damage processes and $\mathrm{pH}$ changes suggesting environments sensitive to the corrosion of steel materials [4]. Early RFID sensor investigation has been performed by Mita and

*jerlynch@umich.edu; phone 1734 615-5290; http://www-personal.umich.edu/ jerlynch/index.html

Sensors and Smart Structures Technologies for Civil, Mechanical, and Aerospace Systems 2007, edited by Masayoshi Tomizuka, Chung-Bang Yun, Victor Giurgiutiu, Proc. of SPIE Vol. 6529 652919, (2007) - 0277-786X/07/\$18 - doi: 10.1117/12.715826 
Takahira $[5,6]$ where they have fabricated a prototype $2.16 \mathrm{MHz}$ RFID capacitive peak strain sensor based on two concentric aluminum pipes separated by a dielectric material. Upon installing these peak strain sensors at the base of a seven-story base-isolated building at Keio University, sensor performance coincides with data obtained from a laser displacement transducer [7]. Similarly, Todd [8] extends Mita and Takahira's work by proposing an RFID peak strain sensor fabricated with MEMS processes. Upon applied strain, conductive metal blocks sandwiching a dielectric material will change in capacitance, thereby retaining maximum peak strain. More recently, Chuang et al. [9] demonstrate a high-resolution passive wireless strain sensor exhibiting great linearity up to $130 \mu \mathrm{m} / \mathrm{m}$ strains. The $2.4 \mathrm{GHz} \mathrm{RF}$ (radio frequency) cavity wireless sensor exhibits a quality factor of greater than 2000 with a sensitivity of $2.6 \mathrm{kHz}$ resonant frequency shift per microstrain. Finally, Jia and Sun [10] develop a novel passive thick film strain sensor by incorporating poly(vinyl fluoride) (PVDF) with an interdigital capacitor to enhance strain sensitivity of the wireless sensor. Although only tested in tensile strain, their prototype sensor exhibits near-linear change in resonant frequency up to $18,000 \mu \mathrm{m} / \mathrm{m}$ strains. On the other hand, Bernhard et al. [11] have developed a $2.4 \mathrm{GHz}$ embedded wireless sensor to detect concrete corrosion processes via acoustic emissions; their sensor can identify the loss of interfacial bond strength and reduction in reinforcing steel cross-sectional area. Instead of using an active acoustic transducer to sense corrosion, Simonen et al. [12] have designed a small-footprint RFID sensor by implementing an external switch exposed to its surrounding concrete as the corrosion sensing element. Using different gauge wires for monitoring different thresholds of corrosion, they have successfully demonstrated dramatic characteristic frequency shifts between initial and corroded states.

In this study, a unique multifunctional material in which strain and corrosion transduction mechanisms can be encoded is derived from the nanotechnology domain. Since the discovery of carbon nanotubes (CNT) in 1991 [13], researchers have expanded their applications to include nanocomposites [14], nanoelectronics [15], and a variety of sensing transducers $[16,17]$. It has been shown in previous studies that layer-by-layer (LbL) carbon nanotube-polyelectrolyte thin films can be engineered to exhibit changes in resistance due to applied strain or when in contact with different $\mathrm{pH}$ environments [18]. Extensions of this prior work are made in this study to realize a passive wireless RFID sensor by fabricating coil-patterned LbL nanocomposites whose constituents include single-walled carbon nanotubes (SWNT), gold nanoparticles (GNP), and various polyelectrolytes (PE). First, RFID validation studies performed with SWNT-PE strain and $\mathrm{pH}$ sensors coupled with traditional copper-wire coil antennas are presented. SWNT-PE strain and pH sensors are subjected to applied tensile-compressive cyclic loading (to $\pm 10,000 \mu \mathrm{m} / \mathrm{m}$ ) and to different $\mathrm{pH}$ buffers (1 to 10$)$ while corresponding changes in the RFID sensor's resonant frequency and bandwidth are measured. Finally, a SWNTGNP nanocomposite thin film is patterned into a coil antenna to verify potential thin film wireless communication capabilities.

\section{RADIO FREQUENCY IDENTIFICATION (RFID)}

In its most basic form, radio frequency identification systems consist of a reader and a remote passive tag circuit where wireless communication and power transmission are achieved through inductive coupling. The reader typically consists of a coil antenna (inductor) and an alternating current (AC) source and is responsible for sending power while receiving data from the tag. As an AC current source of a certain frequency $(f)$ passes through the coil antenna, a magnetic field is generated that causes a corresponding potential (voltage) drop and AC current to form in a tag in close proximity (based on Faraday's Law) [19]. Typically, the induced potential drop in the tag can power onboard electronics, thereby allowing the tag to collect data and transmit the data back to the reader using amplitude (ASK) or frequency modulation (FSK) techniques [19]. In this study, no tag electronics are included; rather, all sensing data will be transmitted back to the reader by changes in the inductive coupling between reader and tag.

\subsection{Reader}

A bare backbone RFID reader consists of a loop antenna (with inductance $L_{R}$ and inherent series resistance $R_{R}$ ) coupled with an AC sinusoidal source. In this study, the Solartron 1260 impedance gain/phase analyzer will be used as the high precision AC source. As the Solartron 1260 impedance gain/phase analyzer passes a monotonic regulated 3V AC signal through the coil antenna, the magnetic field generated can be calculated as shown in equation 1 (assuming circular coil).

$$
H=\frac{I \cdot N \cdot R^{2}}{2 \sqrt{\left(R^{2}+x^{2}\right)^{3}}}
$$




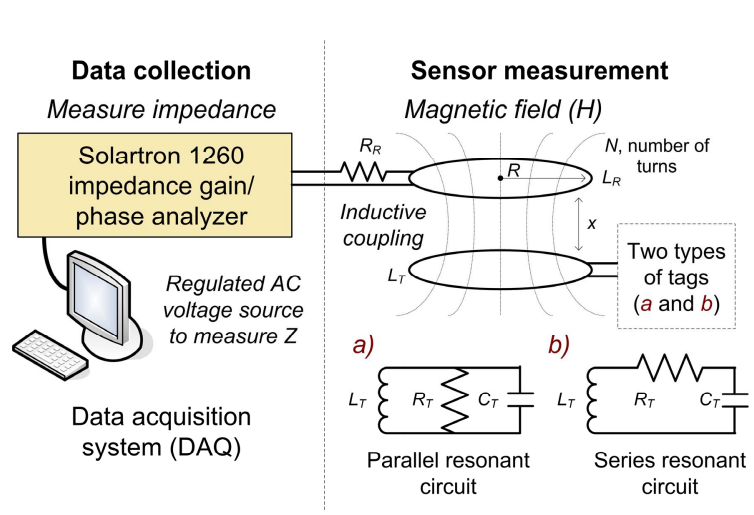

Fig. 1. A schematic illustrating RFID wireless interrogation of a (a) parallel or (b) series resonant tag and data acquisition experimental setup.

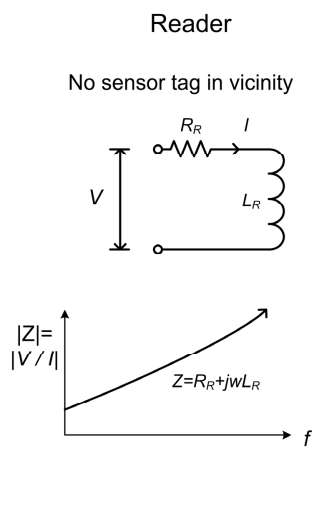

(a)

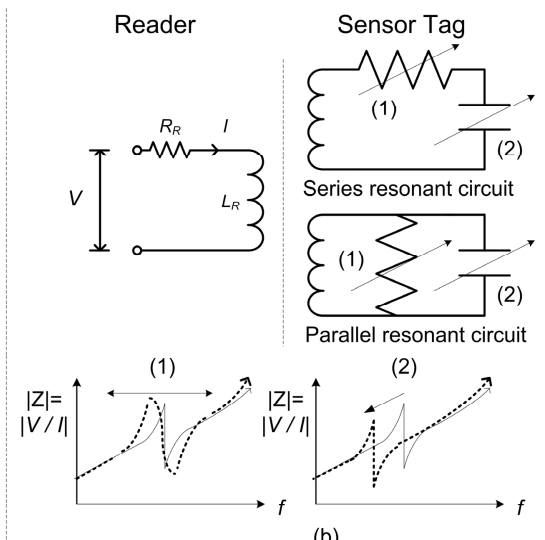

(b)

Fig. 2. (a) A schematic illustrating RFID reader impedance $(Z)$ response when no sensor tag is in the vicinity. (b) When present with a series or parallel resonant circuit, a resistance or capacitance change will cause bandwidth (1) and resonant frequency (2) to change.

where $I$ is the current passing through the coil, $R$ is the antenna radius, $N$ is the number of turns, and $x$ is the readdistance along the central axis of the coil (Figure 1). From equation 1, it can be observed that a tradeoff exists between different size antennas; smaller antennas are capable of generating higher fields at the coil axis, but larger antennas generate higher magnetic fields over larger distances $(x)[19]$.

\subsection{Tag}

Without considering any digital electronic components coupled with the tag circuitry, the simplest RFID tag (or transponder) consists of an inductor coil antenna $\left(L_{T}\right)$ with inherent series resistance $\left(R_{S}\right)$, a resistor $\left(R_{T}\right)$, and a capacitor $\left(C_{T}\right)$ configured as an RLC series or parallel resonant circuit (Figure 1). While different resonant circuit configurations are used for a variety of applications, the resonant frequency of a series and parallel tag will provide maximum current (minimum impedance) or maximum voltage (maximum impedance), respectively [20]. In either case, the defining characteristics of any RFID tag are its resonant frequency $\left(f_{n}\right)$ and bandwidth $(B)$, as shown in equations 2 and 3 respectively.

$$
\begin{gathered}
f_{n}=\frac{1}{2 \pi \sqrt{L_{T} C_{T}}} \\
B_{\text {series }}=\frac{R_{T}}{2 \pi L_{T}} \\
B_{\text {parallel }}=\frac{1}{2 \pi R_{T} C_{T}}
\end{gathered}
$$

While the resonant frequency is independent of tag circuit configuration, calculations of tag bandwidth varies depending on whether it is a series (equation 3a) or parallel resonant circuit (equation 3b).

\subsection{Coupled Reader and Tag System}

When the sensor tag enters the vicinity of the reader, the Solartron 1260 impedance gain/phase analyzer measures the inductively coupled sensor response superimposed onto the impedance of the reader coil (over a certain frequency range) as depicted in Figure 2. Considering only the reader coil antenna without the presence of a sensor tag, the complex impedance $(Z=V / I)$ of the reader can be represented by equation 4 .

$$
Z=R_{R}+j \omega L_{R}
$$


where $\omega$ is the input AC sinusoid's natural cyclic frequency (in $\mathrm{rad} / \mathrm{sec}$ ). However, when present with a sensor tag, the reader's complex impedance response is superimposed by an addition $Z_{T}$ ' term due to inductive coupling (equation 5).

$$
Z=R_{R}+j \omega L_{R}+Z_{T}^{\prime}
$$

In general, irrespective of tag circuit configuration (parallel versus series), one can begin to calculate the equivalent complex impedance of each circuit element in the RLC circuit, namely the inductor along with its inherent series resistance $\left(Z_{L}\right)$, capacitor $\left(Z_{C}\right)$, and resistor $\left(Z_{R}\right)$ as shown in equations $6-8$.

$$
\begin{gathered}
Z_{L}=R_{S}+j \omega L_{T} \\
Z_{C}=\frac{1}{j \omega C_{T}} \\
Z_{R}=R_{T}
\end{gathered}
$$

When present with a series resonant circuit, the total measured impedance at the reader side can be calculated with equation 9 (Figure 2b).

$$
Z=R_{R}+j \omega L_{R}+\frac{k^{2} \omega^{2} L_{R} L_{T}}{Z_{L}+Z_{C}+Z_{R}}
$$

On the other hand, for a parallel resonant circuit, the equivalent impedance is (Figure 2b),

$$
Z=R_{R}+j \omega L_{R}+\frac{k^{2} \omega^{2} L_{R} L_{T}}{1 / Z_{L}+1 / Z_{C}+1 / Z_{R}}
$$

where the first two terms in equations 9 and 10 represent the impedance due to the reader antenna coil alone (as shown in equation 4) while $k$ is the coupling factor (between 0 and 1). In short, the coupling factor qualitatively describes the mutual inductance between the reader and tag coil antennas. Although $k$ can be calculated based on coil radii and read range, the coupling factor is strongly dependent on coil geometry, among other factors. Nevertheless, full coupling $(k=$ 1) can be theoretically achieved when the distance between the reader and tag coils is zero [19].

In this study, irrespective of the strain or $\mathrm{pH}$ sensing transduction mechanism, sensor response is translated into resonant frequency shifts or bandwidth changes (equations 2 and 3 and Figure 2b). For example, when a sensor coupled to a series resonant circuit exhibits a change in capacitance, its resonant frequency changes (equation 2), but its bandwidth remains fixed (equation 3a). A schematic detailing the RFID reader response when the sensor resistance and capacitance changes is presented in Figure 2 (for both series and parallel resonant tag circuits). While it is easier to identify resonant frequency shifts in the frequency-domain, bandwidth change can be accurately captured via numerical model-fitting of experimental results.

\section{LAYER-BY-LAYER SENSOR FABRICATION}

Homogeneous thin film carbon nanotube sensors are fabricated via a layer-by-layer template-assisted self-assembly technique where oppositely charged species are deposited one monolayer at a time (Figure 3) [18, 21, 22]. In short, by sequentially dipping a charged substrate in polycationic and polyanionic solutions, the weak van der Waals and electrostatic force interaction allow one to deposit uniform monolayers of controlled thickness. In addition, the flexibility of the LbL assembly technique allows one to tailor multilayer thin films with specific functionalities simply by controlling the type of polyelectrolyte and nanomaterials used during fabrication [18]. In this study, three different types of multilayer films are fabricated with specific sensing properties, namely (1) capacitive strain sensitive films, (2) $\mathrm{pH}$ sensitive films using a conductive polymer, and (3) carbon nanotube-gold nanoparticle conductive films for inductive coupling in RFID wireless communications (Figure 4).

To begin, the layer-by-layer self-assembly technique is realized by dipping a negatively-charged substrate (e.g. glass, silicon, poly(ethylene terephthalate) (PET, 3M), among others) in a polycationic solution to deposit the initial monolayer. In this study, $1.0 \%$ by $w t$. poly(vinyl alcohol) (PVA, Sigma), $1.0 \%$ by $w t$. poly(aniline) (PANI, $M_{w} \approx$ 


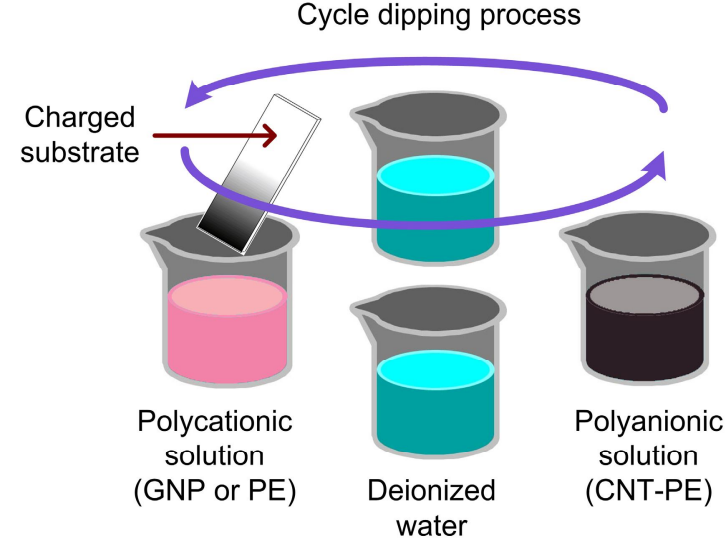

Fig. 3. A schematic of the layer-by-layer self-assembly process.

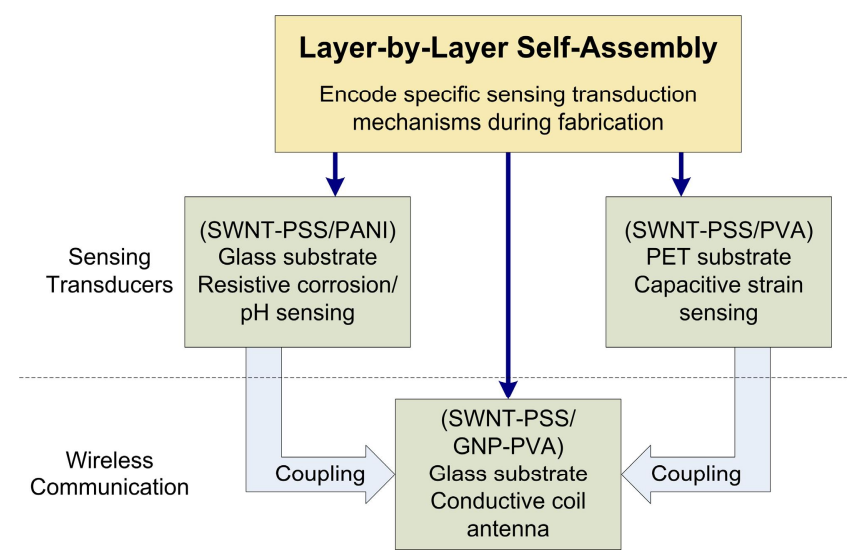

Fig. 4. LbL self-assembly is capable of fabricating multilayer thin films with different sensing transduction mechanisms and high-conductivity films for RFID wireless sensing applications.

100,000, Aldrich), and gold nanoparticles dispersed in 1.0 by wt. PVA are employed to fabricate films of different compositions and sensing functionalities. Upon sufficient rinsing (with Millipore $18 \mathrm{M} \Omega$ deionized water) to remove weakly-adsorbed species and drying (by compressed air) to ensure higher quality film fabrication, the substrate is then dipped in a polyanionic solution to deposit the next monolayer. Typically, single-walled carbon nanotubes suspended in a $1.0 \%$ by $w t$. poly(sodium 4-styrene sulfonate) (PSS, $M_{w} \approx 1,000,000$, Sigma) or a $1.0 \%$ by $w t$. sodium dodecyl sulfate (SDS, $M_{w} \approx 288.38$, Sigma-Aldrich) solution is employed as the negatively charged LbL solution. After completion of rinsing and compressed air drying, the aforementioned process describes one cycle of the LbL multilayer film assembly technique to yield a one-bilayer thin film. This process is repeated $n$ times to produce homogeneous thin films of desirable thicknesses (denoted as $(\mathrm{A} / \mathrm{B})_{n}$, where $\mathrm{A}$ and $\mathrm{B}$ represent the oppositely charged species, and $n$ represents the number of bilayers [22]).

\section{EXPERIMENTAL RESULTS AND DISCUSSION}

\subsection{Wireless Strain Sensor Validation}

Design of the capacitive strain sensor begins by fabricating (SWNT-PSS/PVA) ${ }_{100}$ LbL thin films upon a PET substrate $\left(0.127 \mathrm{~mm}\right.$ thick). By assembling (SWNT-PSS/PVA) ${ }_{100}$ thin films on both sides of the substrate, a SWNT-PE parallel plate capacitor is formed (denoted as SWNT-on-PET). Due to PET's high Poisson's ratio ( $c a$ 0.4), an applied tensile or compressive strain will significantly change sensor dimensions, thus increasing or decreasing the parallel-plate capacitance, respectively (Figure 5). When coupled with a $235 \mu \mathrm{H}$ inductor coil antenna and a $1.2 \mathrm{M} \Omega$ resistor in a parallel resonant tag circuit configuration, a change in capacitance will cause an inversely proportional change in resonant frequency (equation 2).

Validation of strain sensor capacitance, and thereby resonant frequency shift, due to applied strain is accomplished by applying a one-cycle tensile-compressive cyclic load pattern $( \pm 10,000 \mu \mathrm{m} / \mathrm{m})$ to the specimens. Prior to mechanical loading, SWNT-on-PET capacitive strain sensors are affixed onto a PVC (poly(vinyl chloride) Type I) bar using CN-E epoxy (Tokyo Sokki Kenkyujo). Upon sufficient drying time (6 hours), each specimen is mounted in an MTS-810 load frame (Figure 6) where a tensile-compressive cyclic load pattern is applied at $2,500 \mu \mathrm{m} / \mathrm{m}$ strain increments. At each strain increment, the applied strain from the MTS-810 load frame is held for approximately $5-10$ min to allow the Solartron 1260 impedance gain/phase analyzer to measure the coupled impedance with the RFID-based sensor and to determine the sensor's resonant frequency change of the capacitive wireless strain sensor (Figure 1).

To measure the strain sensor tag response, the Solartron 1260 impedance gain/phase analyzer applies a monotonic $3 \mathrm{~V}$ $\mathrm{AC}$ sinusoid over a range of frequencies to measure the complex impedance of the reader inductively coupled with the 
Unstrained capacitive strain sensor
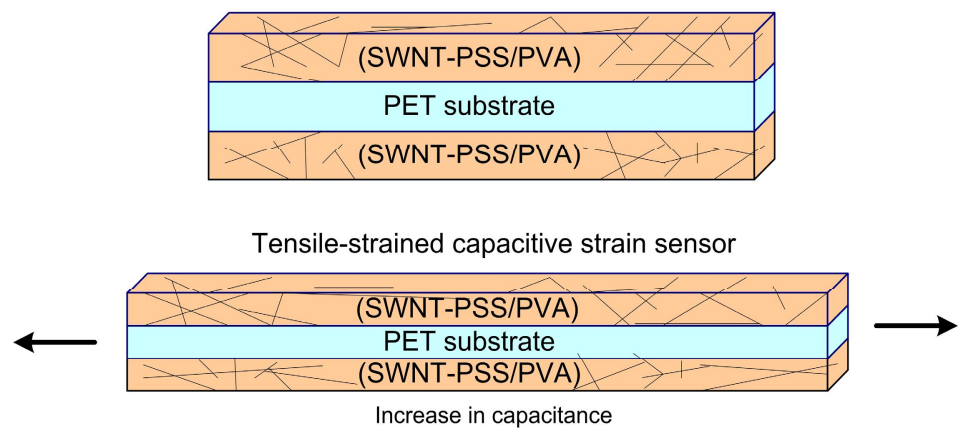

Fig. 5. Conceptual illustration showing how strain affects thin film dimensions that lead to capacitance change.

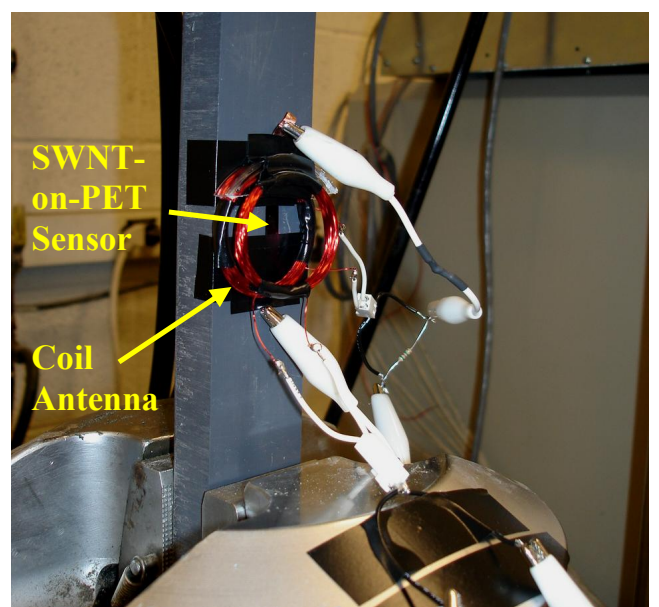

Fig. 6. SWNT-on-PET capacitive strain sensor (epoxy-bonded to a PVC bar) in a parallel resonant circuit configuration loaded by the MTS-810 load frame.

strain sensor tag. Since the SWNT-on-PET capacitive strain sensor behaves similar to a parallel-plate capacitor, its capacitance can be estimated by equation 11 .

$$
C_{S W N T-o n-P E T}=\frac{\varepsilon_{r} \varepsilon_{0} w L}{g}\left(1+\varepsilon_{s}\right)
$$

where $\varepsilon_{r}$ is the relative dielectric permittivity of PET, $\varepsilon_{0}$ is the permittivity of air $\left(\varepsilon_{0}=8.854 \cdot 10^{-12} \mathrm{~F} / \mathrm{m}\right), \mathrm{w}($ width $)$ and $L$ (length) are the dimensions of the parallel-plate capacitor, $g$ is the thickness of the PET thin film substrate $(0.127 \mathrm{~mm})$, and $\varepsilon_{s}$ is the applied strain. From equation 11, it is obvious that as the SWNT-on-PET film is under applied tension (in the direction of the length of the sensor), the Poisson's effect causes the width and thickness of the material to decrease while elongating the length. Assuming that PET is a homogeneous and isotropic material, then Poisson's ratio is the same in all directions and only the change in dimension along the axis of the applied load affects the capacitance. Thus, with increasingly applied tension, sensor capacitance will increase and the opposite is true for specimens under compression.

Upon applying a one-cycle tensile-compressive cyclic load pattern to the SWNT-on-PET capacitive strain sensor, the experimental results as measured by the Solartron 1260 impedance gain/phase analyzer are shown in Figure 7. As mentioned by Simonen et al. [12], the resonant frequency can be identified by observing the inflection point on the impedance magnitude-frequency plot (Figure 7) or equivalently, a dip in the phase angle. Although the differential frequency between each impedance measurement is set very small $(\Delta f=1 \mathrm{kHz})$, interpolation between experimental data at each applied strain is required to quantify a more precise value for resonant frequency. Processed experimental results shown in Figure 8a confirm the change in resonant frequency as a function of applied tensile and compressive strains (between $\pm 10,000 \mu \mathrm{m} / \mathrm{m})$. By pre-determining the tag coil inductance $\left(L_{T}\right)$, SWNT-on-PET capacitance can be calculated from experimental results to produce Figure $8 \mathrm{~b}$. Theoretically, the shift in resonant frequency is non-linear (a $1 / \sqrt{ }\left(1+\varepsilon_{\mathrm{s}}\right)$ relationship), as can be identified by plugging in equation 11 into equation 2 to yield equation 12 .

$$
f_{n}=\frac{1}{2 \pi \sqrt{L_{T} \varepsilon_{r} \varepsilon_{0} w L\left(1+\varepsilon_{s}\right) / g}}
$$

Since the degree of non-linearity is small, one can estimate the sensitivity of this wireless capacitive strain sensor by numerically fitting a linear least-squares best-fit line to extract the slope or the sensor sensitivity. The sensitivity of the capacitive strain sensor can be estimated as $0.427 \mathrm{~Hz} / \mu \mathrm{m}-\mathrm{m}^{-1}$. Although the estimated strain sensitivity is low, a more significant resonant frequency shift can be achieved by changing sensor dimensions (increasing length and width or decreasing thickness) to increase film capacitance (equation 11). It should be noted that the tag inductance should be 


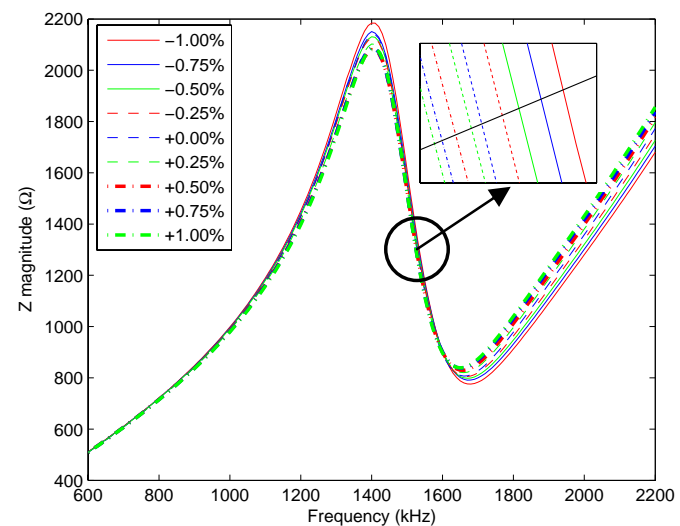

Fig. 7. Experimental RFID reader response of SWNT-on-PET capacitive strain sensor under one-cycle tensilecompressive cyclic loading to $\pm 10,000 \mu \mathrm{m} / \mathrm{m}$ (inset shows zoomed in plot near the resonant frequency).
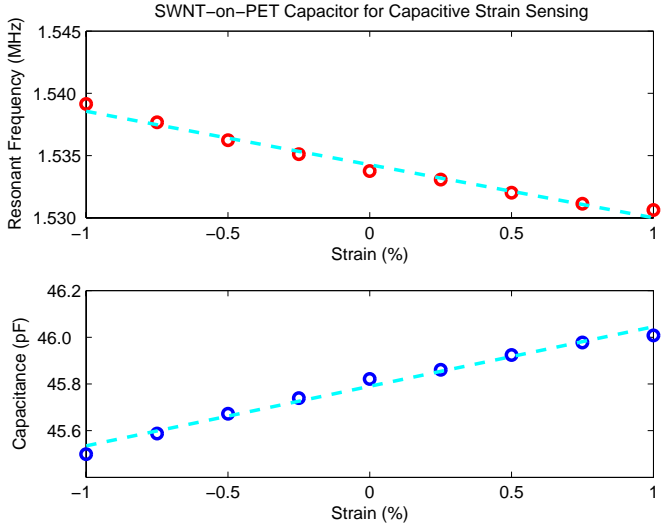

Fig. 8. (a) Resonant frequency shift of SWNT-on-PET capacitive strain sensor under one-cycle tensilecompressive cyclic loading to $\pm 10,000 \mu \mathrm{m} / \mathrm{m}$; (b) corresponding SWNT-on-PET capacitance change.

adjusted to achieve the same resonant frequency. Thus, by increasing capacitance, the relative change in capacitance will be larger thereby causing a more significant shift in resonant frequency due to strain.

\subsection{Wireless pH Sensor Validation}

Prior to validating the (SWNT-PANI/PVA $)_{50}$ thin film wireless $\mathrm{pH}$ sensor's performance, a preliminary study is conducted to measure resistance change as the sensor is exposed to different $\mathrm{pH}$ buffer solutions (ranging from $\mathrm{pH} 1-$ 10). Using an Agilent 64401A digital multimeter, thin film resistance is sampled at $1 \mathrm{~Hz}$ to characterize film resistance evolution and $\mathrm{pH}$ sensitivity. From Figure 9a, the experimental time history plot suggests dramatic resistance change with increasing $\mathrm{pH}$ buffer solutions. The sensitivity $\left(S_{p H}\right)$ of the sensor and its degree of linearity can be extracted as shown in Figure $9 \mathrm{~b}$ and calculated via equation 13 (where $A$ represents thin film area in $\mathrm{cm}^{2}$ ).

$$
S_{p H}=\frac{\Delta R / A}{\Delta p H}
$$

From Figure 9b, and upon normalization by film area, it has been determined that the (SWNT-PANI/PVA) $)_{50}$ thin film $\mathrm{pH}$ sensor is roughly characterized by a $19.9 \mathrm{k} \Omega-\mathrm{cm}^{-2} / \mathrm{pH}$ linear sensitivity (results similar to those reported in [18]).

To proceed onto validating the RFID $\mathrm{pH}$ sensor performance, the $\mathrm{pH}$ sensitive (SWNT-PSS/PVA) $)_{50}$ thin film is included in a parallel resonant circuit to measure bandwidth change as a function of $\mathrm{pH}$ buffer solution. In this case, a $11 \mathrm{~mm} 7$ $\mathrm{mm}(\mathrm{SWNT}-\mathrm{PANI} / \mathrm{PVA})_{50}$ thin film acts as the $\mathrm{pH}$-sensitive resistor (on the order of 10-100 $\mathrm{k} \Omega$ ) while connected in

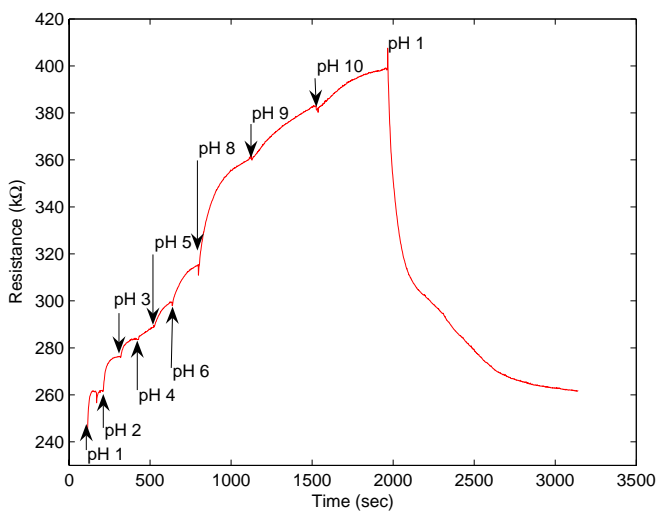

(a)

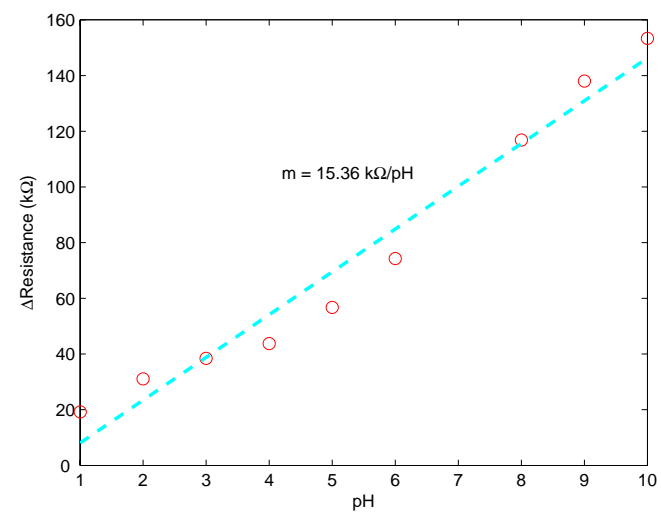

(b)

Fig. 9. (a) Time history plot of (SWNT-PSS/PANI) $5_{50}$ thin film resistance due to different $\mathrm{pH}$ buffer solutions. (b) Linearity plot plotting change of resistance as a function of applied $\mathrm{pH}$ buffer solution indicating $\mathrm{pH}$ sensor sensitivity is $15.36 \mathrm{k} \Omega / \mathrm{pH}$. 


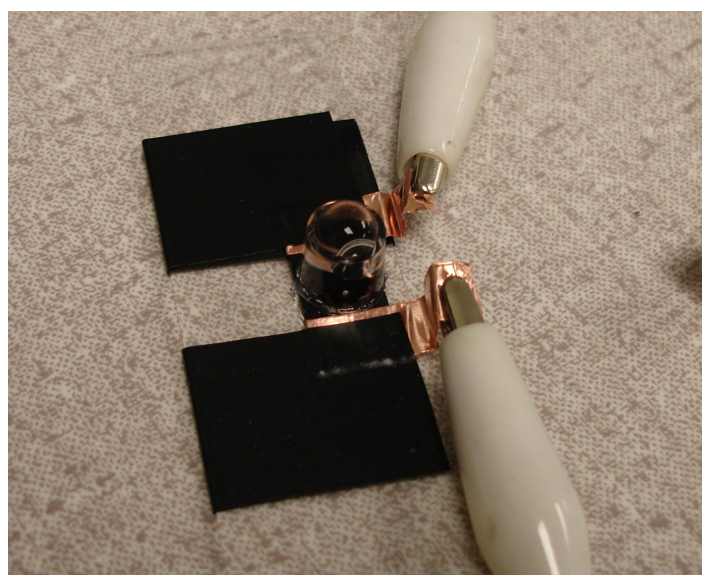

Fig. 10. (SWNT-PSS/PANI) $)_{50}$ thin film $\mathrm{pH}$ sensor (as part of a parallel resonant circuit) with plastic well mounted for pipetting $\mathrm{pH}$ buffer solutions.

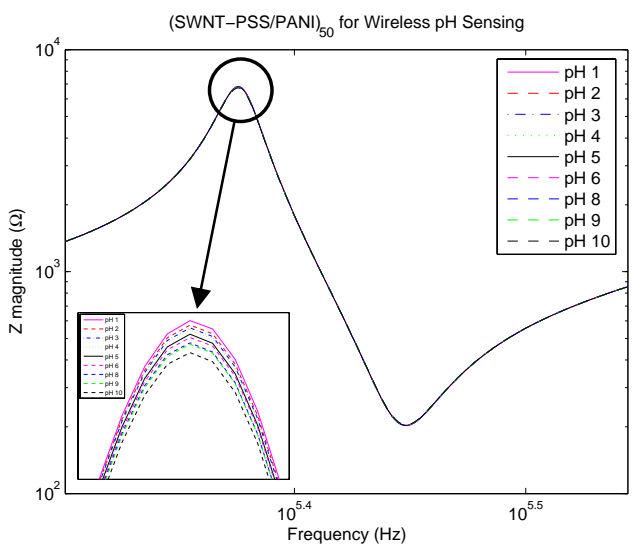

Fig. 11. Impedance magnitude-frequency response as measured by the Solartron 1260 reader to capture the wireless $\mathrm{pH}$ sensor performance with different $\mathrm{pH}$ buffer solutions.

parallel to a $2,100 \mu \mathrm{H}$ inductor coil and a $220 \mathrm{pF}$ capacitor. To control the amount of $\mathrm{pH}$ solution exposed to the $(\text { SWNT-PANI/PVA) })_{50}$ thin film, a plastic well is mounted on top of the thin film via high vacuum grease (Dow Corning) (Figure 10). Buffer solutions $(1 \mathrm{~mL})$ ranging from $\mathrm{pH} 1-10$ are pipetted into the plastic well while the Solartron 1260 impedance gain/phase analyzer wirelessly measures the frequency response of the parallel resonant $\mathrm{pH}$ sensor tag, where the experimental data is plotted in Figure 11.

Since the (SWNT-PANI/PVA) 50 thin film electrochemical sensing transduction mechanism is based on resistance change, the parallel resonant circuit seeks to identify bandwidth change due to applied $\mathrm{pH}$ buffer solutions (equation $3 \mathrm{~b}$ ). Bandwidth change is inherently more difficult to characterize when compared with resonant frequency shifts. As a result, the experimentally derived impedance curve is numerically fitted for every $\mathrm{pH}$ solution to obtain the equivalent circuit parameters. Using a stochastic simulated annealing model updating algorithm to minimize the least squares difference between experimental data and equation 10, the individual tag circuit parameters (particularly $R_{T}$ and $C_{T}$ ) are extracted to calculate system bandwidth (equation 3b). A typical model-fitted overlay is shown in Figure 12, indicating accurate modeling between theory and experiment. As a result, sensor bandwidth is plotted as a function of $\mathrm{pH}$ as shown in Figure 13; based on equation $3 \mathrm{~b}$, it is no surprise that bandwidth decreases nonlinearly with increasing $\mathrm{pH}$ buffer solution. Since the change is nonlinear, it is difficult to identify $\mathrm{pH}$ bandwidth sensitivity; rather, it can be seen from Figure 13 that the tag bandwidth changes from 270 to $25 \mathrm{~Hz}$ as $\mathrm{pH}$ scales from 1 to 10 .

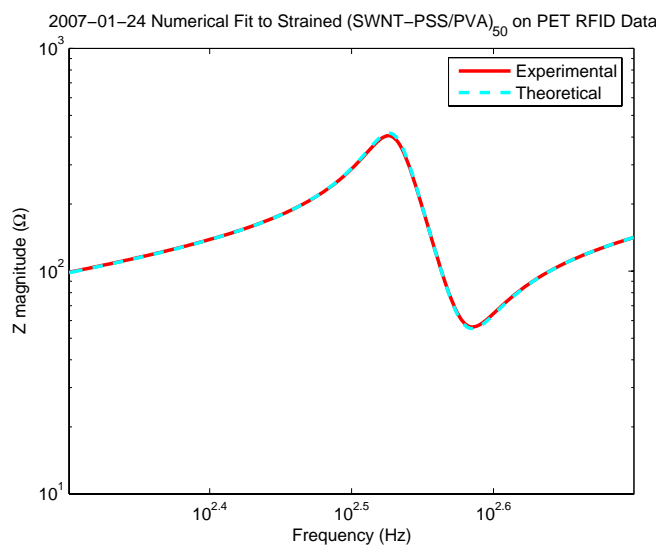

Fig. 12. Validation of stochastic simulated annealing modelfitting between theoretical and experimental RFID reader response indicates good fit.

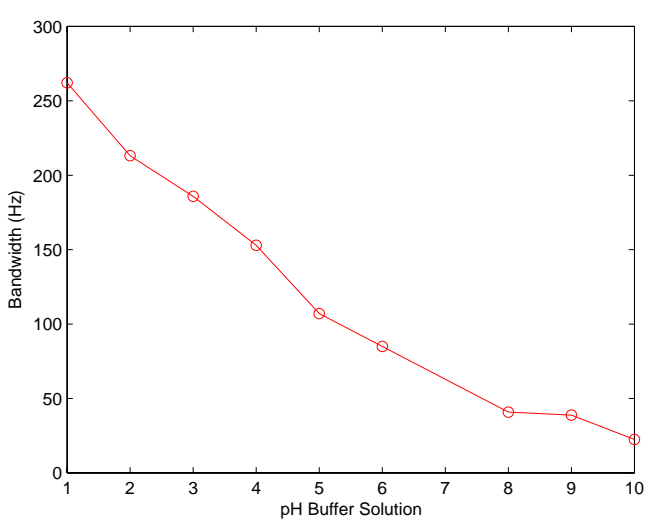

Fig. 13. (SWNT-PSS/PANI) $)_{50}$ thin film parallel resonant circuit shows considerable bandwidth change due to applied $\mathrm{pH}$ buffer solution. 


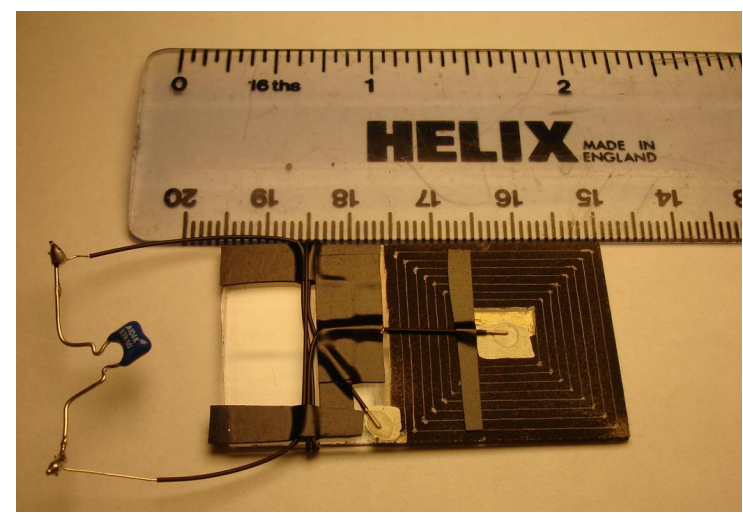

Fig. 14. Picture of patterned (SWNT-SDS/GNP-PVA) $)_{50}$ thin film coil antenna connected to a $0.1 \mu \mathrm{F}$ capacitor to form a series resonant circuit.

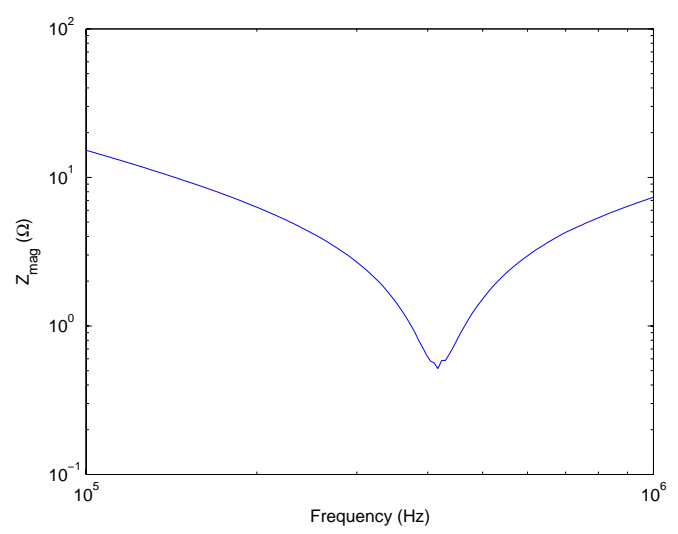

Fig. 15. Experimental RFID reader response collected from (SWNT-SDS/GNP-PVA) thin film coil antenna coupled with a $0.1 \mu \mathrm{F}$ capacitor.

\subsection{Conductive Carbon Nanotube-Gold Nanocomposite for RFID Inductive Coupling}

In order to realize a CNT-based LbL inductor coil antenna, the conductivity of the patterned film needs to be high to achieve low-bandwidth RFID systems. Liu et al. [23] have reported that ionic self-assembled GNP-PE thin films exhibit the same order of magnitude conductivity as bulk gold ( $\mathrm{Au}$ ) metals. Due to the moderate resistivity of typical LbL CNTPE thin films ([18]), a CNT-PE inductor coil antenna will be limited by its high series resistance. As seen from equation 3a, a high series resistance will increase the system bandwidth dramatically, rendering any wireless communication futile. In compensation, carbon nanotubes are dispersed in SDS to improve CNT deposition during LbL fabrication; since CNTs exhibit near-ballistic-type electronic transport properties, increasing CNT deposition and solution concentration can yield more conductive thin films ([24-26]). In an attempt to dramatically increase LbL SWNT-PE thin film conductivity, gold nanoparticles $\left(\mathrm{HAuCl}_{4}\right.$ from Alfa AESAR) are incorporated with the aforementioned SWNT-PE fabrication methodology (Section 3).

As opposed to using polyelectrolyte solutions such as PVA (for strain sensing) or PANI (for $\mathrm{pH}$ sensing), gold nanoparticles are dispersed in a $1.0 \%$ by wt. PVA solution for enhancing film conductivity. Preparation of this cationic solution begins by dissolving $1.0 \mathrm{mg} / \mathrm{mL} \mathrm{HAuCl}_{4}$ in a $1.0 \%$ by wt. PVA solution followed by the addition of $0.1 \mathrm{M}$ sodium borohydride $\left(\mathrm{NaBH}_{4}\right)$ solution. The LbL technique continues by utilizing GNP-PVA and SWNT-SDS solutions to fabricate multilayer thin films on a glass substrate (denoted as (SWNT-SDS/GNP-PVA) ${ }_{n}$ thin films). Finally, upon completion of film fabrication, (SWNT-SDS/GNP-PVA) ${ }_{n}$ thin films undergo high temperature annealing $(\mathrm{T}=350 \mathrm{C})$ for $10 \mathrm{~min}$ to further enhance film conductivity. Preliminary results have shown that (SWNT-SDS/GNP-PVA) ${ }_{n}$ nanocomposite films exhibit 4 to 5 times lower resistance than (SWNT-PSS/PVA) ${ }_{n}$ thin films (as reported in [18]).

Upon completion of (SWNT-SDS/GNP-PVA) $)_{50}$ thin film fabrication, the multilayer nanocomposites are etched into 25 $\mathrm{mm} \times 25 \mathrm{~mm}$ squares and patterned into a coil antenna configuration via high precision mechanical cutting (Figure 14). Since thin film conductivity is high (low resistance), a series resonant tag circuitry is formed by connecting a $0.1 \mu \mathrm{F}$ capacitor with the patterned (SWNT-SDS/GNP-PVA) 50 thin film. Electrical contacts between the capacitor and thin film are made by drying silver paste (Ted Pella) over single-strand wires connected to the series capacitor. Since the inductor coil is patterned via mechanical cutting, only a low number of turns are achievable on the $25 \mathrm{~mm}$ x $25 \mathrm{~mm}$ sample space (seven to eight turns as seen in Figure 14). Instead of wirelessly interrogating the (SWNT-SDS/GNP-PVA) $)_{50}$ antenna, the Solartron 1260 impedance gain/phase analyzer is connected to the tag circuit to test for series resonant conditions. Since a series resonant circuit is formed by connecting the inductor coil (with some inherent series film resistance) to a $0.1 \mu \mathrm{F}$ capacitor, the impedance-frequency response plot (or Bode plot) should follow equation 14.

$$
Z=Z_{L}+Z_{C}+Z_{R}
$$

where $Z_{L}, Z_{C}$, and $Z_{R}$ are described in equations 6,7 , and 8 respectively. From equation 14, one can deduce that resonance will occur at minimal impedance where a significant dip should be observed in the Bode plot as seen from experimental data in Figure 15. It can be observed from Figure 15 that the nanocomposite thin film RFID tag exhibits resonance at $f_{n} \approx 417 \mathrm{kHz}$ as measured by the Solartron 1260 impedance gain/phase analyzer. 


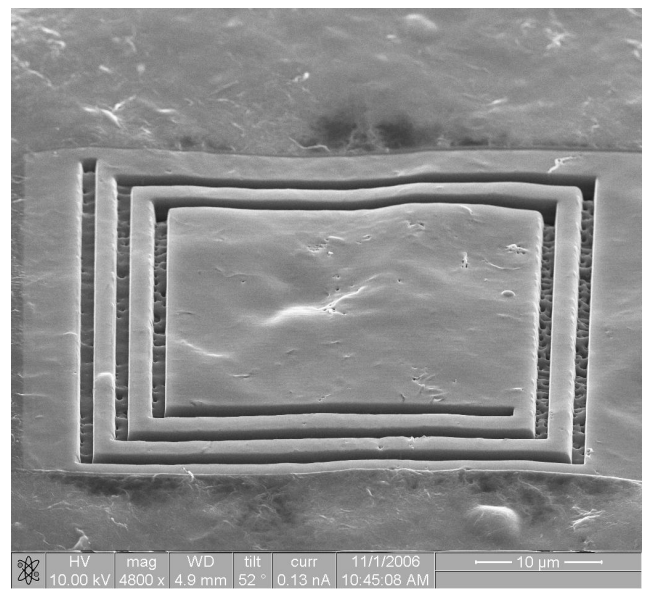

Fig. 16. SEM image showing (SWNT-PSS/PVA) $)_{50}$ thin film prototype coil antenna patterned using FIB at the University of Michigan EMAL facility.

Although the bandwidth of the system is large, results indicate promise for developing a multifunctional carbon nanotube-based RFID sensor. Realization of wireless communication can be achieved by further reducing the series resistance (bandwidth) of the antenna coil. Moreover, since only a few turns (seven to eight) are etched on the thin film, the inductance of the coil antenna is low; however, by patterning more turns, one can achieve greater inductance as shown in equation 15 [20].

$$
L_{S W N T} \cong \frac{0.31(a N)^{2}}{6 a+9 h+10 b} \quad(\text { in } \mu \mathrm{H})
$$

where $a$ is the average radius of the coil (cm), $N$ is the number of turns, $b$ is the winding thickness (cm), and $h$ is the winding height $(\mathrm{cm})$. As a first step to improve the inductance of these thin films, various specimens have been patterned into coil antennas via a focused ion beam (FIB) scanning electron microscope (SEM) at the University of Michigan Electron Microbeam Analysis Laboratory (UM EMAL). A sample film SEM image (measuring $40 \mu \mathrm{m}$ x 40 $\mu \mathrm{m}$ ) is shown in Figure 16. In the near future, lithographic processes will be employed to pattern dense coil structures onto large surface areas. Thus, a large number of coils can be achieved in a larger area, thereby increasing thin film inductance to enhance wireless communication capabilities.

\section{CONCLUSIONS}

In this study, a layer-by-layer carbon nanotube-based nanocomposite thin film strain and $\mathrm{pH}$ wireless RFID sensor is presented. Validation studies conducted indicate that the fabricated SWNT-on-PET capacitive wireless strain sensor exhibits shifts in resonant frequency due to capacitance change. The change in resonant frequency due to an applied tensile-compressive cyclic loading to $\pm 10,000 \mu \mathrm{m} / \mathrm{m}$ suggests a sensor sensitivity of $0.427 \mathrm{~Hz} / \mu \mathrm{m}-\mathrm{m}^{-1}$. Although the sensitivity is lower than those reported in the literature, higher sensitivity can be achieved by varying sensor dimensions to increase capacitance. On the other hand, (SWNT-PSS/PANI) $)_{50}$ thin films are employed as $\mathrm{pH}$ sensors; their resistance change to applied $\mathrm{pH}$ buffer solution (pH 1 to 10 ) is dramatic, with a sensitivity approximately $19.9 \mathrm{k} \Omega-\mathrm{cm}^{-2} / \mathrm{pH}$. When coupled with a parallel resonant circuit for wireless sensing, bandwidth change from 270 to $25 \mathrm{~Hz}$ is observed when the (SWNT-PSS/PANI $)_{50}$ thin films are subjected to $\mathrm{pH} 1-10$ buffer solutions.

Finally, using carbon nanotube-gold nanocomposites, highly conductive thin films have been fabricated and patterned into coil antennas for wireless communication. In this case, gold nanoparticles are used to increase the conductivity of the thin films (5 times higher than SWNT-PSS/PVA films) for low-bandwidth RFID systems. Preliminary results suggest that the patterned coil antenna, when coupled with a capacitor, indeed behaves as a series resonant circuit with potential for wireless communications. However, due to its moderate resistance and low number of turns, alternative methods need to be employed to reduce the bandwidth and to increase inductance respectively. To enhance inductance, 
work has begun to pattern coil antennas via FIB at the UM EMAL facility. In the near future, lithographic processes will be utilized to pattern dense coil structures for enhancement of inductance and sensor read range.

\section{ACKNOWLEDGEMENTS}

This research is supported by the National Science Foundation (Grant Number CMS - 0528867). The authors would like to express their gratitude to Professor Victor Li and the ACE-MRL group for allowing us to use the MTS-810 load frame during the strain-testing phase of this study. The authors would also like to thank the UM EMAL staff for technical assistance and training with FIB patterning. Furthermore, the authors would like to express their gratitude to Dr. Nadine Wong Shi Kam for providing assistance with processing PANI.

\section{REFERENCES}

1. C. R. Farrar, "Historical Overview of Structural Health Monitoring," Lecture Notes on Structural Health Monitoring using Statistical Pattern Recognition, Los Alamos Dynamics, Los Alamos, NM (2001).

2. J. P. Lynch and K. J. Loh, "A Summary Review of Wireless Sensors and Sensor Networks for Structural Health Monitoring," Shock and Vibration Digest, 38(1), 1-38 (2006).

3. J. P. Lynch, Y. Wang, K. J. Loh, J. H. Yi, and C. B. Yun, "Performance Monitoring of the Geumdang Bridge using a Dense Network of High-Resolution Wireless Sensors," Smart Materials and Structures, 15, 1-15 (2006).

4. P. A. M. Basheer, K. T. V. Grattan, T. Sun, A. E. Long, D. McPolin, and W. Xie, "Fiber Optic Chemical Sensor System for Monitoring $\mathrm{pH}$ Changes in Concrete," Proceedings of SPIE - Advanced Environmental, Chemical, and biological Sensing Technologies II, 5586, 144-153 (2004).

5. A. Mita and S. Takahira, "Health Monitorig of Smart Structures Using Damage Index Sensors," Proceedings of SPIE - Smart Structures and Materials, 4696, $92-99$ (2002).

6. A. Mita and S. Takahira, "A Smart Sensor Using a Mechanical Memory for Structural Health Monitoring of a Damage-Controlled Building," Smart Materials and Structures, 12(2), 204-209 (2003).

7. A. Mita and S. Takahira, "Damage Index Sensor for Smart Structures," Structural Engineering and Mechanics, 17(3-4), 331-346 (2004).

8. M. Todd, "Different Approaches Towards Deploying SHM Sensor Arrays: Wireless Communications with Autonomous Vehicle Interrogation," Proceedings of the $5^{\text {th }}$ International Workshop on Structural Health Monitoring, 1594-1601 (2005).

9. J. Chuang, D. J. Thomson, and G. E. Bridges, "Embeddable Wireless Strain Sensor Based on Resonant RF Cavities," Review of Scientific Instruments, 76, 094703/1-094703/7, (2005).

10. Y. Jia and K. Sun, "Thick Film Wireless and Powerless Strain Sensor," Proceedings of SPIE - Smart Structures and Materials, 6174, 61740Z/1-61740Z/11 (2006).

11. J. T. Bernhard, K. Hietpas, E. George, D. Kuchma, and H. Reis, “An Interdisciplinary Effort to Develop a Wireless Embedded Sensor System to Monitor and Assess Corrosion in the Tendons of Prestressed Concrete Girlders," IEEE Topical Conference on Wireless Communication Technology, 241-243 (2003).

12. J. T. Simonen, M. M. Andringa, K. M. Grizzle, S. L. Wood, and D. P. Neikirk, "Wireless Sensors for Monitoring Corrosion in Reinforced Concrete Members," Proceedings of SPIE - Smart Structures and Materials, 5391, 587-596 (2004).

13. S. Iijima, "Helical Microtubules of Graphitic Carbon," Nature, 354, 56-58 (1991).

14. E. T. Thostenson and T-W Chou, "Aligned Multi-Walled Carbon Nanotube-Reinforced Composites: Processing and Mechanical Characterization,” J. Phys. D: Apl. Phys., 35, L77-L80 (2002).

15. K. Tsukagoshi, N. Yoneya, S. Uryu, Y. Aoyagi, A. Kanda, Y. Ootuka, and B. W. Alphenaar, "Carbon Nanotube Devices for Nanoelectronics," Physica B, 323, 107-114 (2002).

16. J. Kong, N. R. Franklin, C. Zhou, M. G. Chapline, S. Peng, K. Cho, and H. Dai, "Nanotube Molecular Wires as Chemical Sensors," Science, 287, 622-625 (2000).

17. J. R. Wood, Q. Zhao, M. D. Frogley, E. R. Meurs, A. D. Prins, T. Peijs, D. J. Dunstan, and H. D. Wagner, "Carbon Nanotubes: From Molecular to Macroscopic Sensors," Physical Review B, 62, 7571-7575 (2000).

18. K. J. Loh, J. Kim, J. P. Lynch, N. W. S. Kam, and N. A. Kotov, "Multifunctional Layer-by-Layer Carbon NanotubePolyelectrolyte Thin Films for Strain and Corrosion Sensing," Smart Materials and Structures, 16, 429-438 (2007). 
19. K. Finkenzeller, RFID Handbook Fundamentals and Applications in Contactless Smart Cards and Identification, Wiley, West Sussex, England 2003.

20. Y. Lee, "RFID Coil Design," Microchip AN678, 1-18 (1998).

21. G. Decher, "Fuzzy Nanoassemblies Toward Layered Polymeric Multicomposites," Science, 277, 1232-1237 (1997).

22. N. A. Kotov, "Ordered Layered Assemblies of Nanoparticles," MRS Bulletin, 26, 992-997 (2001).

23. Y. Liu, Y. Wang, and R. O. Claus, "Layer-by-Layer Ionic Self-Assembly of Au Colloids into Multilayer Thin-Films with Bulk Metal Conductivity," Chemical Physics Letters, 298, 315-319 (1998).

24. M. Zhang, L. Su, and L. Mao, "Surfactant Functionalization of Carbon Nanotubes (CNTs) for Layer-by-Layer Assembling of CNT Multi-Layer Films and Fabrication of Gold Nanoparticle/CNT Nanohybrid," Carbon, 44, 276-283 (2006).

25. K. J. Loh, J. P. Lynch, B. S. Shim, and N. A. Kotov, "Identification of Layer-by-Layer Fabrication Parameters for Optimizing Multilayer Carbon Nanotube-Polymer Composite Strain Sensors," Journal of Intelligent Materials Systems and Structures (accepted 2007).

26. R. H. Baughman, A. A. Zakhidov, and W. A. de Heer, "Carbon Nanotubes - The Route Towards Applications," Science, 297, 787-792 (2002). 